\title{
KONFERENZBEITRÄGE
}

Aufsätze zur Tagung „(Neo-)Avantgarde in der tschechischen und slowakischen Literatur/Kunst“ am 7. Mai 2010, abgehalten in Wien aus Anlass des 75. Geburtstages von Pavol Winczer

WIENER SLAVISTISCHES JAHRBUCH, Band 57/2011, 7-20

(C) 2011 by Österreichische Akademie der Wissenschaften, Wien

STEFAN SIMONEK

\section{Offenheit: Ein zentrales Diskursmoment in Programmschriften der westslawischen historischen Avantgarde}

Im Rahmen des folgenden, knapp gehaltenen Beitrags soll der Versuch unternommen werden, drei zentrale Programmtexte der westslawischen historischen Avantgarde aus der ersten Hälfte der zwanziger Jahre des zwanzigsten Jahrhunderts über die in den Manifestationen der Avantgarde durchgehend zu beobachtende, paradoxe Gleichzeitigkeit von diskursiver Offenheit und Geschlossenheit zueinander in Relation zu stellen. Diese komplexe Verbindung einander eigentlich widersprechender Momente mag vielleicht mit der simultanen Teilhabe dieser Programmtexte an jenen zwei grundlegenden ästhetischen Modellen korrespondieren, die der russische Theoretiker Jurij M. Lotman als Ästhetik der Identität („èstetika toždestva“) bzw. als Ästhetik der Gegenüberstellung (,èstetika protivopostavlenija“) bezeichnet hat und die sich jeweils über die Bestätigung und Befestigung einer künstlerischen Norm einerseits sowie über deren Brechung und Überschreitung andererseits definieren (Lotman 1973: 432-437). Manifest- und Programmtexte stehen als solche chronologisch gesehen ja immer am Anfang einer sich gerade neu etablierenden und durchsetzenden künstlerischen Strömung, sie programmieren diese gleichsam in die Zukunft hinein und funktionieren in dieser Hinsicht als Vor-Wurf, als utopische Projektion in der ursprünglichen Bedeutung dieses Begriffs (vgl. Grübel 1998: 162 f.). Als solche formulieren diese Texte in thesen- wie regelhafter Weise die zentralen programmatischen Anliegen der neuen Richtung und erheben von daher einen normierenden ästhetischen Anspruch, der sich unter Lotmans Definition einer „Ästhetik der Identität" subsumieren lässt. In Widerspruch dazu unterliegen die Programmtexte der historischen Avantgarde der zehner und zwanziger Jahre des vergangenen 
Jahrhunderts aber ganz grundsätzlich dem mit großem rhetorischen Aufwand inszenierten Pathos von Innovation, forcierter Beschleunigung und radikaler Überwindung des Alten; unter diesem Gesichtspunkt wiederum können sie als integrale Momente einer auf die Spitze getriebenen „Ästhetik der Gegenüberstellung“ interpretiert werden, die sich nicht nur in den westeuropäischen, sondern auch in den slawischen Literaturen spätestens Ende des 18. bis Mitte des 19. Jahrhunderts in Sentimentalismus und Romantik als dominierendes künstlerisches Modell durchgesetzt hat.

Die Manifeste der historischen slawischen Avantgarde sind also Schwellentexte nicht nur auf einer zeitlichen Achse, sondern zusätzlich auch in Bezug auf ihre prinzipielle ästhetische Modellierung zwischen Normbruch und Normbestätigung, mithin also zwischen einem affirmierenden und einem destruierenden Pol. Zusätzlich dazu oszillieren sie vielleicht nicht zuletzt eben aufgrund dieser grundlegenden Ambivalenz noch zwischen den Gegensätzen von Offenheit und Geschlossenheit; letztere manifestiert sich in den Programmtexten auf verschiedene Weise. Ganz grundsätzlich streben diese Texte danach, die eigenen künstlerischen Positionen zu legitimieren und jene der Vorgänger bzw. konkurrierender zeitgenössischer Gruppierungen in verschiedenster Weise zu devaluieren: Das diskursive Bestreben, die eigenen Positionen rhetorisch als die einzig gültigen in Anschlag zu bringen, belegt anschaulich die Mechanismen, die zur Genese der von Michel Foucault beschriebenen „Ordnung des Diskurses" führen und resultieren in der von Foucault hervorgehobenen Bestrebung, sich „,im Wahren“ zu befinden (Foucault 1997: 24 f.) und deshalb konsequenterweise alle übrigen Positionen abzuwerten. Ermöglicht wird dies nicht zuletzt durch den Umstand, dass die entsprechenden Programmtexte sehr oft in der ersten Person Plural abgefasst sind, d. h. im Namen einer größeren Gruppe, wohingegen die zurückgewiesenen und als obsolet punzierten antagonistischen Positionen in der zweiten oder dritten Person Plural präsentiert werden - man sitzt also im Namen der eigenen ästhetischen Parameter (,wir“) über seine Gegner unmittelbar zu Gericht (,,ihr“) oder aber lagert diese in den Bereich des Abwesenden und damit Unverbindlichen aus (,sie“).

Ganz im Kontrast zu diesen vielfältigen diskursiven Mechanismen von Abgrenzung und Ausschließung sind die Programmtexte der Avantgarde gleichzeitig aber von einem grundlegenden Zug der Offenheit geprägt, auf die 1974 bereits Peter Bürger in seiner vieldiskutierten Theorie der Avantgarde implizit hingewiesen hat. Wenn Bürger hier die historische Avantgarde als die erste künstlerische Bewegung bezeichnet, der ohne jede Einschränkung die Gesamtheit der künstlerischen Mittel aus allen davor gelagerten Epochen zur Verfügung steht (Bürger 2007: 23), so mag hinter der gewählten Begrifflichkeit (im Sinne einer Verfügungsgewalt oder einem umstandslosen Indienstnehmen) zwar erneut ein Zug von Geschlossenheit präsent sein; nichtsdestoweniger eröffnet Bürgers Ansatz ganz primär eine Reihe von Anschlussmöglichkeiten in Richtung einer Kontextualisierung der avantgardistischen Programmtexte. Offenheit würde sich in diesem Zusammenhang einmal als pro- 
grammatisches literarisches Motiv in den Texten selbst, dann in ästhetischer Hinsicht als die von der Avantgarde proklamierte Ausweitung des Aktionsradius von Kunst und in einem interkulturellen Sinn schließlich als Bezugnahme auf internationale künstlerische Referenzgrößen manifestieren, die die slawische Avantgarde in einen breiter dimensionierten europäischen Rahmen hineinstellen.

All diesen Aspekten von Offenheit soll nun über einen Vergleich je eines zentralen Programmtextes aus der tschechischen, der slowakischen und der polnischen Avantgarde nachgegangen werden, die von ihren Veröffentlichungsdaten lediglich drei Jahre auseinander liegen und sich damit in einer Konstellation relativer zeitlicher Nähe zueinander befinden: Karel Teiges erste poetistische Programmschrift mit dem Titel Poetismus erschien 1924 in der Brünner Zeitschrift „Host“ und stellte das eigentliche Manifest der neuen künstlerischen Richtung dar, auch wenn der Text selbst anders als der vier Jahre später in der Prager „Revue Devětsilu“ veröffentlichte Beitrag Manifest poetismu den Begriff „Manifest“ selbst nicht im Titel führte (Teiges späteres Manifest des Poetismus wiederum unterliegt aufgrund seines Umfangs und seiner im Wesentlichen resümierenden und nicht projektiven Argumentationsweise nicht der Poetik des Manifestes; der Umstand, dass Teige hier aber den früheren Text des Jahres 1924 rückblickend als Manifest bezeichnet, ${ }^{1}$ erlaubt es, eben diesen früheren Text als eigentliche Absichtserklärung in Sachen der neuen künstlerischen Richtung zu betrachten). Ebenfalls im Jahr 1924 erschien in Nr. 6 der Zeitschrift „Mladé Slovensko“, dem Organ des slowakischen Studentenverbandes, Ladislav Novomeskýs knapp gehaltene Programmschrift mit dem Titel Náhl'ady, die lediglich rund zwei Seiten stark ist. Bruno Jasieńskis mit „Krakau, am 20. April 1921“ datierte polnische Programmschrift Manifest w sprawie natychmiastowej futuryzacji życia schließlich erschien im Krakauer Almanach der Futuristen mit dem Titel „Jednodniówka futurystów“ bereits drei Jahre früher im Juni 1921. ${ }^{2}$ In ihrer Gesamtheit bieten diese drei Schriften wohl einen einigermaßen repräsentativen Querschnitt durch die Programmatik der westslawischen Avantgarde zu Beginn der zwanziger Jahre.

In allen drei Programmtexten ist die zuvor erwähnte, sich nach innen hin gruppenbildend einschließende und nach außen hin gegen konkurrierende Strömungen wie auch gegen die literarischen Vorgänger hin abgrenzende erste Person Plural als Instanz des Sprechens präsent (wenn auch in abgestufter Deutlichkeit); dieser

1 „Dva roky nato, na jaře 1924, je publikován můj manifest poetismu [...]“ (Teige 1966: 325 f.). - Unter diesem Gesichtspunkt erweist sich Oleg Sus' Behauptung, wonach der erste Programmtext Teiges bald nach Erscheinen und bis herauf in die Gegenwart zum „ersten Manifest“ des Poetismus umgetauft wurde (Sus 1964: 367), als unzutreffend, nahm Karel Teige diese Neubenennung 1928 doch selbst vor.

2 Im Original wichen (entsprechend der hier auch auf die orthographische Ebene durchschlagenden Verfremdungsästhetik des Futurismus) die Schreibung der Titel sowohl des Almanachs selbst als auch des darin enthaltenen Manifestes in einigen Positionen ab, vgl. die Ansicht des Titelblattes der „Jednodniówka futurystów“ in Stern 1969: 48. 
„Wir“-Diskurs kann freilich keinesfalls als Errungenschaft der historischen Avantgarde bezeichnet werden, er ist in strukturell völlig homologer Konstellation bereits in den Programmschriften der slawischen Moderne, etwa bei Zenon PrzesmyckiMiriam oder Stanisław Brzozowski, präsent - in ihren Absichtserklärungen Nasze zamiary (1887) und My młodzi (1902) ist die erste Person Plural ebenso bereits im Titel gegeben wie im 1898 veröffentlichten, zu Przesmycki-Miriam titelgleichen Beitrag Naše težnje des Kroaten Milivoj Dežman-Ivanov. Analoges gilt klarerweise auch für das insgesamt von zwölf Personen unterzeichnete, von Josef Svatopluk Machar verfasste Manifest der tschechischen Moderne von Oktober 1895; auch hier werden die künstlerischen wie politischen Forderungen natürlich im Namen der Unterzeichner erhoben, vgl.: „Chceme všeobecné hlasovací právo“ (Česká moderna 1950: 363).

In Ladislav Novomeskýs Náhl’ady nun erweist sich der „Wir“-Diskurs als durchgehend dominant, beginnend vom unmittelbaren Anfang der Programmschrift „Stúpame vzdušnýmy krokmi po živote“ (Novomeský 1970: 16) bis hin zum letzten Satz des Textes „Myšlienkam radosti slová ohnivé, ktoré pritisnú na prsia naše celý vel'ký svet“ (17). Dabei wird im Verlauf des von ihrem rhetorischen Aufwand und ihrer gedrängten Ausdrucksweise her an ein Prosagedicht erinnernden Absichtserklärung an keiner Stelle deutlich, in wessen Namen der Autor eigentlich spricht; eine klar konturierte Zugehörigkeit zu einer literarischen Gruppierung geht aus dem Text selbst jedenfalls nicht hervor. Etwas anders gelagert ist der Fall bei Jasieńskis Manifest, das analog zu den Ansichten Novomeskýs ebenfalls von einem markant ausformulierten Diskurs des „Wir“ geprägt ist, der hier freilich eindeutig mit einer bestimmten Gruppenzugehörigkeit verbunden ist, nämlich jener zu den polnischen $\mathrm{Fu}$ turisten. Die Formulierung „My, futuryści polscy“ (Jasieński 1969: 208) funktioniert in der verkürzten Form „My, futuryści“ als ein mehrfach rekurrierendes, strukturbildendes Element des Textes $(211,214)$. Einen Mittelweg zwischen Novomeský und Jasieński geht schließlich Karel Teige: Seine erste poetistische Programmschrift aus dem Jahr 1924 ist zwar ebenfalls in der ersten Person Mehrzahl abgefasst, freilich wird die Gruppenzugehörigkeit bei weitem nicht derart in den Vordergrund gerückt, wie dies bei Jasieński der Fall ist. Formulierungen wie „to, co nazveme POETISMEM“ (Teige 1966: 121) oder „Relativisté, jsme přesvědčeni o skryté iracionalitě“ (123) repräsentieren in Teiges Text bereits die radikalsten Belegstellen für diese Form eines diskursiv generierten Sich-Abschließens als Distinktionsmerkmal gegenüber anderen, konkurrierenden künstlerischen Gruppierungen. ${ }^{3}$

Ähnlich wie alle drei hier referierten Programmtexte über die Verwendung der ersten Person Plural diskursive Geschlossenheit generieren, verhält es sich in umge-

${ }^{3}$ Oleg Sus verweist in Bezug auf Teiges erstes poetistisches Manifest eben auf diese limitierte Abgeschlossenheit, wenn er vermerkt: „Střetá se tu kolektivismus plus rozumovost s touhou po individuálním vyžití a citovým vybitím [...]“"(Sus 1964: 379). 
kehrter Richtung nun auch mit dem Phänomen der Offenheit im Sinne einer angestrebten Erweiterung des Bereichs künstlerischer Aktivität auch jenseits der immer stärker als Beengung empfundenen tradierten Institutionen. Die für die gesamte historische Avantgarde charakteristische Tendenz, die traditionellen und statischen Institutionen einer bürgerlich geprägten elitären Hochkultur, wie etwa Museum oder Konzertsaal, abzuwerten und im Gegensatz dazu beweglichere Formen der Populärkultur, wie Zirkus oder Kabarett, aufzuwerten und den Schauplatz der Kunst gewissermaßen aus den geschlossenen Räumlichkeiten der Hochkultur hinaus auf die Straße zu verlagern, tritt in allen drei Programmtexten deutlich hervor. Dies lässt die westslawische Avantgarde mindestens in dieser Hinsicht als Kontinuum einer breiter gefassten, gesamteuropäischen Strömung erscheinen. ${ }^{4}$

Bei Bruno Jasieński wird dieser Gedanke in drei aufeinander folgenden Schritten inszeniert: Zuerst erfolgt im Zeichen des Annihilationsgestus in Richtung der kanonisierten künstlerischen Wertsetzungen die (möglicherweise auch von den Manifesten des russischen Kubo-Futurismus inspirierte ${ }^{5}$ ) Forderung nach einer tabula-rasaKonstellation, die in ihrer Leere und Offenheit erst den Raum für die Entfaltung zukünftiger, utopisch gesetzter kultureller Wertsetzungen ermöglicht: Um die Postamente freizubekommen, die Plätze zu säubern und denjenigen, die da kommen werden, Platz zu schaffen, wollen die Futuristen mit Hilfe von Schubkarren die Mumien all der Mickiewicz' und Słowackis von den Plätzen und Straßen fortschaffen (Jasieński 1969: 209). ${ }^{6}$ Diese provokative Abwertung der eigenen literarischen Tradition, die sich im Text des Manifestes unter Anderem in der Kleinschreibung und Pluralsetzung der beiden polnischen Nationaldichter äußert (,,mumie mickiewiczów i słowackich“), findet in einem zweiten Schritt dann ihre logische Fortsetzung in der Forderung nach mehr Sonne („Więcej słońca“; 210); letztere wiederum ist in ihrem utopischen Anspruch für die futuristischen Programmtexte insgesamt ebenso charakteristisch wie die darauf folgende Ablehnung von Schirmen, Hüten und Zylindern und der Aufruf, barhaupt zu gehen (ebd.).

Als weiterer Schritt zur Gewinnung von Offenheit nach der Zurückweisung der beengenden Traditionen und der gleichermaßen beengenden Kopfbedeckungen folgt bei Jasieński dann die auch graphisch hervorgehobene Forderung an die Künstler,

${ }^{4}$ Zur Einbettung der westslawischen Manifeste in einen allgemeineuropäischen Horizont vgl. die von Wolfgang Asholt und Walter Fähnders edierte Anthologie von Programmtexten der europäischen Avantgarde (Asholt - Fähnders 1995).

$5 \mathrm{Zu}$ Parallelen zwischen der poetischen Praxis Jasieńskis und jener der russischen KuboFuturisten, insbesondere Vladimir Majakovskijs, vgl. Stern 1968: 16 ff., 30-35 bzw. Drews 1983: $67 \mathrm{f}$.

6 Vgl. dazu auch die von den russischen Kubo-Futuristen Burljuk, Majakovskij, Kručenych und Chlebnikov in ihrem auf Dezember 1912 datierten Manifest Poščečina obščestvennomu vkusu erhobene Forderung, die hoch kanonisierten Autoren aus der eigenen Tradition des 19. Jahrhunderts, namentlich Puškin, Dostoevskij und Tolstoj, vom Dampfer der Gegenwart hinunter zu stoßen (Poščečina 1924: 99). 
auf die Straße hinaus zu treten („Artyści na ulicę!“; ebd.), und die avantgardistische Absage an die traditionellen Räume der Hochkultur, wie Konzertsaal, Ausstellung oder Museum, denen der Autor den Vorwurf des Elitären macht. Als positiven, utopisch gesetzten Gegenentwurf einer räumlich wie zeitlich entgrenzten und jedermann zugänglichen Kunst führt Jasieński dann die Idee von ununterbrochen andauernden Konzerten und Lesungen in Zügen, Straßenbahnen, Parks und Passagen sowie auf öffentlichen Plätzen an (210 f.). Damit kombiniert er die für die Avantgarde kennzeichnenden Momente von Technik und Beschleunigung mit den ebenso typischen Versuchen, den Produkten der Kunst (mit Walter Benjamin gesprochen) ihre traditionelle Aura zu nehmen. ${ }^{7}$ Nicht mehr der Ausstellungswert, sondern der Gebrauchswert der Kunst soll nun im Vordergrund stehen, und vor diesem Hintergrund mag es nur konsequent erscheinen, wenn Jasieński die Diskurselemente von räumlicher Offenheit und Demokratisierung (mithin also Öffnung) der Kunst in seinem Manifesttext auch ineinander verschränkt: Inmitten einer Abfolge kürzerer Sätze, in denen die Präsenz der Kunst auf der Straße insgesamt dreimal durchvariiert wird, findet sich eine wiederum auch graphisch hervorgehobene Aussage, wonach jedermann Künstler sein könne („Każdy może być artystą“; 211).

In Karel Teiges drei Jahre später veröffentlichtem poetistischen Manifest ${ }^{8}$ findet sich eine auffällig analog gehaltene Argumentationsabfolge, die freilich kaum einer direkten Rezeption des polnischen Programmtextes als vielmehr der Teilhabe an einem gesamteuropäischen Diskursmuster avantgardistischer Positionen geschuldet sein dürfte. Ganz ähnlich wie im Aufruf des polnischen Futuristen werden die angestrebte Öffnung der Kunst und die Ablehnung der tradierten kulturellen wie akademischen Institutionen auch in diesem tschechischen Text über räumliche Parameter generiert und im Verlauf des Manifestes insgesamt dreimal variiert. Teige positioniert die Schönheit der Welt, der sich die neue Richtung des Poetismus zutiefst verpflichtet fühlt, nicht in den geschlossenen Räumlichkeiten von Kathedralen und Galerien, sondern draußen auf den Straßen und in den Parks: „Neusídila se v katedrálách či galeriích; ven do ulic, [...] v ozvěžující zeleni parků [...] našla svi̊j domov“ (Teige 1966: 122). Dieser auch von Jasieński artikulierte Gedanke durchzieht in abgewandelter Weise dann auch den weiteren Text Karel Teiges.

So werden einige Seiten später die muffigen Arbeitszimmer und Ateliers als Negativfolie gegen einen herrlich duftenden Park als eigentlichen Ort der Kunst in Stellung gebracht (123) und zwei Absätze darauf die Atmosphäre des Lebens (ein Zentralbegriff des Poetismus) mit jener von Arbeitszimmer, Bibliothek und Museum also klassischen Räumlichkeiten intellektueller wie künstlerischer Kontemplation -

${ }^{7}$ Vgl. Benjamins kanonische Definition der Aura als ,einmalige Erscheinung einer Ferne, so nah sie sein mag“ in seinem zentralen Aufsatz Das Kunstwerk im Zeitalter seiner technischen Reproduzierbarkeit (Benjamin 2003: 15).

8 Zur Programmatik des Poetismus insgesamt vgl. Hansen-Löve 1975: 106-116; zu Karel Teiges Position gegenüber dem Poetismus vgl. Zusi 2004. 
konfrontiert (124). Im letzten Satz seiner Programmschrift koppelt Teige dann ähnlich wie Jasieński die positivwertige Semantisierung von Offenheit mit der Ablehnung der Tradition, wenn er der Literatur, die von den Poetisten zum alten Eisen geworfen wird (und die damit ein ähnliches Schicksal erleidet wie Mickiewicz und Słowacki im polnischen Programmtext), Ausflüge, belebte Boulevards und Kurortpromenaden gegenüberstellt (128). Die Ansichten Ladislav Novomeskýs - der sich gerade in diesen Jahren intensiv mit den poetistischen Innovationen seiner tschechischen Kollegen auseinandersetzte - fügen sich über die Betonung von Offenheit mehr oder weniger nahtlos an die zwei bisher referierten Programmtexte an und belegen die zentrale Relevanz dieser Kategorien. Wie schon Jasieński und Teige, so arbeitet auch der slowakische Lyriker mit der Metapher der Straße, die jeweils am Beginn der zwei Abschnitte des Textes in programmatischer Weise auftaucht: Die allgemeiner gehaltene Feststellung „Cesty sú najpodivnejšie“ (Novomenský 1970: 16) wird dann in einem zweiten Schritt auf das kollektive Subjekt bezogen und so in den „Wir-Diskurs“ integriert, wenn es heißt: „Po tej ceste nechodíme za vyhraneným ciel'om“"(17).

Neben der Metapher der Straße als Sinnbild für den erweiterten Handlungsraum avantgardistischer Kunst findet sich bei Bruno Jasieński zusätzlich noch die Metapher der weit geöffneten Fenster und Türen, die über das programmatisch hervorgehobene Merkmal der Offenheit zum einen mit dem Motiv der Straße korrespondiert, die daneben aber noch eine zusätzliche diachrone, über die drei hier referierten Programmtexte hinausreichende Achse eröffnet. Diese Achse ist nun in dreifacher Hinsicht von Relevanz: Erstens führt sie zu später veröffentlichten Texten Teiges und Novomeskýs und zeigt analog zur Metapher der Straße die enge wechselseitige Verbindung der Avantgardetexte auf einer motivischen Ebene; daneben eröffnet sie über das Moment der (auch kulturell verstandenen) Öffnung eine Anschlussstelle zu der noch zu erörternden interkulturellen Offenheit in den Programmtexten der Avantgarde und schließlich führt sie in den Bereich der Moderne zurück und belegt so die Kontinuität zwischen Moderne und Avantgarde mindestens in dieser Hinsicht.

In Jasieńskis futuristischem Manifest wird die Forderung nach einer Öffnung der Fenster und Türen mit dem Innovationsgestus der Avantgarde in Verbindung gebracht, der sich hier gegen die in Polen traditionell einflussreiche religiöse Instanz der Kirche, aber auch gegen die alltägliche, gewohnte Lebensweise der Bevölkerung richtet: „Trzeba otworzyć na oścież wszystkie drzwi i okna, niech wyweje ten swąd piwnic i kościelnego kadzidła, którym od dziecka uczyli was oddychać“ (Jasieński 1969: 209). In Karel Teiges zweiter, 1928 veröffentlichter poetistischer Programmschrift Manifest poetismu ${ }^{9}$ wird die Metapher der geöffneten Fenster dagegen als

${ }^{9}$ Vgl. zum Kontext dieser zweiten poetistischen Programmschrift Teiges Bydžovská 1999; zu den Unterschieden zwischen Teiges erster und zweiter poetistischer Programmschrift vgl. Brousek 1975: 176 ff., zu den Positionen der beiden Texte vgl. Müller 1978: 32-42. 
kulturelle Öffnung der tschechischen Kultur hin zu Europa verstanden, wobei die eigene tschechische Tradition im Zeichen des avantgardistischen Antitraditionalismus ab-, die Zugehörigkeit der eigenen kulturellen Wertsetzungen zu einer größer konzipierten europäischen Einheit dagegen aufgewertet wird. ${ }^{10}$ Teige bemerkt in diesem Zusammenhang:

Naši předchůdci otevřeli okna do Evropy. Vyvodili jsme z dokonale internacionální povahy moderní civilizace ten důsledek, že jsme opustili provinciální a regionální horizonty nacionální a státní př́slušnosti, odcizili jsme se dějinám literatury české a zřekli se dědictví českého malířství (Teige 1966: 328).

Ausgehend von dieser Positionierung Teiges, die über die Metapher der in Richtung Europa hin geöffneten Fenster verläuft, lassen sich nun weitere der historischen Avantgarde in den westslawischen Literaturen verpflichtete Belegstellen anführen, in denen eine analoge Motivik zu beobachten ist. So führen die geöffneten Fenster etwa wieder zu Ladislav Novomeský zurück, der seinen 1935 veröffentlichten Gedichtband eben mit Otvorené okná überschrieb und (mindestens die uns zugängliche, zweite Auflage des Bandes aus dem Jahre 1964) mit dem Gedicht Hoci epilóg abschloss, wo das Titel gebende Motiv am Beginn der vierten Strophe in der Zeile „Tak som stál opretý v tom okne otvorenom“ (Novomeský 1971: 156) auf das lyrische Subjekt des Gedichtes bezogen wird. In umgekehrter Richtung verläuft eine diachrone Verbindungslinie zur tschechischen Avantgarde vor dem Ersten Weltkrieg, also zur so genannten „Generation von 1914“, die sich über das Motiv des geöffneten Fensters als analog zur Programmatik der Poetisten der zwanziger Jahre erweist. In Stanislav Kostka Neumanns an die Manifeste der Futuristen sowohl italienischer wie russischer Prägung erinnernden Programmschrift Otevřená okna, die am 9. August 1913 in den „Lidové noviny“ erschien, werden die Vorgänger der eigenen tschechischen Kultur im Kontext der Moderne (etwa in der Gestalt Josef Svatopluk Machars, Julius Zeyers oder Alfons Muchas) zugunsten des befreiten Wortes und zugunsten von Expressionismus, Kubismus und Orphismus - also aktuellen internationalen Strömungen - abgewertet (vgl. Drews 1983: 83).

Peter Drews hat in seiner instruktiven Studie zu den Beziehungen der slawischen Avantgarde mit dem Westen auf Übereinstimmungen zwischen Neumanns Programmschrift und der tschechischen Moderne um das Jahr 1895 herum aufmerksam gemacht (84), und tatsächlich lassen sich ungeachtet der Tatsache, dass in Neumanns Text mit Machar ausgerechnet der Verfasser des Manifestes Česká moderna zurückgewiesen wird, gerade über den Titel Otevřená okna Kontinuitäten zwischen Teige und Neumann auf der einen und zentralen Repräsentanten der Moderne auf der anderen Seite herstellen; der Umstand, dass diese analog zu Ladislav Novomeskýs Gedichtband Otvorené okná aus dem Jahre 1935 wiederum aus dem rein natio-

${ }^{10}$ Zur europäischen Dimension von Teiges künstlerischen Aktivitäten vgl. Fabian 2001. 
nalen Bereich der tschechischen Literatur in die Programmatik des Jungen Polen und gar der Wiener Moderne überleiten, belegt gleichsam das dem Motiv der geöffneten Fenster gleichsam inhärenten Hang zur Überschreitung nationalliterarischer Grenzen. So findet sich die entsprechende Metapher bei Zenon Przesmycki-Miriam bereits 1887 in dem in der ersten Nummer der Warschauer „Życie“ erschienenen Beitrag Nasze zamiary - hier ist vom Fenster nach Europa die Rede, das eben mit Hilfe der neu gegründeten Zeitschrift weiter aufgestoßen werden sollte als bisher (Przesmycki-Miriam 1973: 185). Wenige Jahre später war es dann Hermann Bahr, der in seiner Programmschrift Die Moderne 1890 auf das Bild der geöffneten Fenster zurückgriff; dabei zeigt sich deutlich, dass zwischen den diskursiven Strategien in der Programmatik von (europäischer) Moderne und Avantgarde auch dann, wenn etwaige genetische Kontaktbeziehungen mit großer Wahrscheinlichkeit auszuschließen sind (wie im Falle Hermann Bahrs und Bruno Jasieńskis), signifikante Analogien bestehen, finden sich doch zentrale Elemente aus Jasieńskis Manifest, wie eben das Öffnen der Fenster [1], die utopisch konnotierte Hinwendung zur Sonne [2] und der tabula-rasa-Gestus, also das „Entrümpeln“ devaluierter, abgelebter kultureller Wertsetzungen [3] in ganz ähnlicher Form bereits bei Bahr: „Wir wollen die Fenster weit öffnen [1], daß die Sonne [2] zu uns komme, die blühende Sonne des jungen Mai. [...] Und mit Jubel und Ehrfurcht wollen wir das Licht grüßen, das zur Herrschaft einzieht in die ausgeräumten Hallen [3]“" (Bahr 1968: 36).

Über die Parameter einer diskursiv produzierten Offenheit, die sich in der programmatischen Forderung nach der Verlagerung der Kunst aus den akademischen Institutionen hinaus auf die Straße und nach ihrer Verfügbarkeit auch für breitere Kreise der Bevölkerung manifestiert, legen Jasieńskis futuristisches Manifest, Teiges poetistische Programmschrift und Novomeskýs Ansichten also Züge einer Kontinuität offen, die letztlich in einer gesamteuropäischen Poetik der historischen Avantgarde der zehner und zwanziger Jahre fußt. Umso signifikanter scheint der Umstand, dass sich unter der Perspektive einer anders gelagerten Erscheinungsform von Offenheit - nämlich der Anbindung an ein supranationales literarisches Kontinuum durch die Nennung internationaler künstlerischer Referenzgrößen in den Programmtexten selbst - ein signifikanter Bruch zwischen den Positionen Jasieńskis, Teiges und Novomeskýs ergibt. Dessen Programmschrift ist in dieser Hinsicht am knappsten zu referieren, da sie - mindestens an der Textoberfläche in Sinne eindeutiger namentlicher Erwähnungen - anders als die Schriften Jasieńskis und Teiges gänzlich ohne Nennung irgendwelcher, sei es slowakischer, sei es internationaler Namen auskommt. Sie enthält sich von daher jeglicher sofort und unmittelbar abzulesender Anbindungen an zeitgenössische Strömungen (dieser punktuelle Befund lässt natürlich keine Rückschlüsse auf Novomeskýs übrige Essays aus jener Zeit zu, in denen sich der Autor nicht nur mit dem tschechischen Poetismus, sondern darüber hinaus auch mit zentralen Vertretern sowohl der west- als auch der osteuropäischen 
Avantgarde, wie etwa mit Guillaume Apollinaire oder Vladimir Majakovskij, beschäftigte $^{11}$ ).

Einen anderen Weg geht Bruno Jasieński in seinem Manifest in Sachen der sofortigen Futurisierung des Lebens: Er verschränkt nämlich die geforderte Öffnung der Kunst in Richtung zur Straße hin mit einem höchst ambivalenten Europa-Diskurs, der keinesfalls auf eine rein affirmative Nachahmung europäischer künstlerischer Vorbilder hin abzielt, eher im Gegenteil: Jasieński konstatiert am Beginn seines Manifestes eine Kulturkrise, die den Westen wie den Osten Europas gleichermaßen betrifft (Jasieński 1969: 208); danach perpetuiert er in der Behauptung, dass sich das polnische Kulturbewusstsein nicht so frei entwickeln konnte wie das der westlichen Staaten (ebd.), jenen schon von den Programmtexten des Jungen Polen (etwa bei Przesmycki-Miriam oder Brzozowski) her bekannten Diskurs der kulturellen Abgeschlossenheit und Rückständigkeit. ${ }^{12}$ Dieser wird wenig später dann jedoch in dem für die historische Avantgarde typischen Überbietungsgestus in sein positivwertiges Gegenteil verkehrt: Wenn das polnische Volk (an das sich das Manifest in der Formulierung Do narodu polskiego schon in seinem Titel wendet) nicht länger das letzte, sondern das erste Volk in Europa sein wolle, dann muss es laut Jasieński aufhören, sich von alten Resten aus der Küche des Westens zu ernähren: „Jeżeli nie chcecie być narodem ostatnim w Europie, a przeciwnie, narodem pierwszym, przestańcie raz karmić się starymi ochłapami z kuchni Zachodu“ (209).

Mit einer gewissen Folgerichtigkeit tauchen in der weiteren Argumentation des Textes dann auch zentrale kulturelle Emanationen aus dem Westen, konkret aus der englischen Literatur, in genau diesem ablehnenden Kontext auf und werden gegen die technischen Errungenschaften der modernen Zivilisation ausgespielt - der Morseapparat etwa ist für Jasieński ein tausendmal wichtigeres Kunstwerk als der Don Juan von Byron (212), und das heutige Theaterpublikum würde seiner Meinung nach angesichts von Macbeth ungeniert gähnen (213). Das bedeutet also, dass die westliche kulturelle Tradition von Jasieński ebenso abgelehnt wird wie die eigene, polnische von Mickiewicz herauf bis zu Przybyszewski, der in dem Manifesttext ebenfalls als Beleg für die Abgelebtheit der literarischen Tradition Erwähnung findet (212). Offenheit in Bezug auf die räumliche Emanzipation der Kunst von ihren über-

11 Peter Drews verweist in diesem Zusammenhang auf die Ähnlichkeit zwischen Novomeskýs Programmtexten mit den aphoristischen Manifesten von Vítězslav Nezval (Drews 1983: 242); zur Positionierung Novomeskýs der tschechischen Literatur gegenüber vgl. Patera 1974, zur Vorbildwirkung der poetistischen Lyrik auf Novomeskýs zweite, 1932 erschienene Gedichtsammlung Romboid vgl. Boden 2005, zur darüber hinausreichenden Positionierung der slowakischen historischen Avantgarde im europäischen Kontext insgesamt vgl. Winczer 2001.

12 Vgl. neben Przesmycki-Miriams bereits zitiertem Bild des Fensters nach Europa in Brzozowskis Deklaration My młodzi etwa das Bild des Zaunes, durch den die polnische Gesellschaft angeblich vom europäischen Kulturleben abgetrennt sei (Brzozowski 1973: 136). $\mathrm{Zu}$ damit korrespondierenden Europa-Konzeptionen in zeitlich parallelen Programmtexten der ostslawischen Moderne vgl. Simonek 2005. 
kommenen Institutionen korrespondiert bei Jasieński also keinesfalls mit interkultureller Offenheit, sondern befindet sich weit eher in einem signifikanten Spannungszustand zu letzterer (wobei andererseits jedoch konstatiert werden muss, dass der Autor über die funktionale Gleichstellung von Mickiewicz, Słowacki, Byron und Shakespeare dann freilich doch, wenn auch gleichsam ex negativo, ein gesamteuropäisches kulturelles Kontinuum generiert).

Als dritte Möglichkeit, die sowohl zu Novomeskýs vollständiger Ausklammerung nationaler wie internationaler Referenzgrößen als auch zu deren pointierter Ablehnung durch Jasieński in Kontrast steht, wäre schließlich das poetistische Manifest Karel Teiges zu erwähnen. Hier korrespondieren beide Erscheinungsformen von Offenheit miteinander und werden zu einem engmaschigen Netzwerk verwoben, das jeweils abwechselnd eine Erscheinungsform des Phänomens hervorhebt - die Forderung nach einem Heraustreten der Kunst aus den Galerien und Museen auf die Straße korrespondiert bei Teige mit einer dicht gesetzten Kette von Namen internationaler Künstler, die für die Entfaltung der europäischen Avantgarde von zentraler Bedeutung gewesen sind und die den tschechischen Programmtext auf diese Weise in einen breiteren, auch über Europa selbst hinausweisenden kulturellen Rahmen hineinstellen. ${ }^{13}$ So zitiert Teige etwa in zustimmender Weise einen Satz von Gustave Flaubert (Teige 1966: 122), er erinnert an Walt Whitman (124) ${ }^{14}$, führt die Namen Boccioni (124), Apollinaire ${ }^{15}$, Marinetti ${ }^{16}$, Picasso und Braque in die Diskussion ein (125) und greift mehrmals auf französische Redewendungen und Begriffe wie etwa „Au revoir! Bon vent, bonne mer! Adieu!“ oder „Être de son temps“ zurück, die unübersetzt direkt in den tschechischen Text hineinmontiert werden und so eine weitere interkulturelle Komponente generieren (125 bzw. 127). ${ }^{17}$ Für den polnischen Futuristen Jasieński dient die diachrone Achse primär dazu, die nationale wie auch die

13 Peter Drews hält in diesem Zusammenhang mindestens in Bezug auf den „Devětsil“ dessen weitgehende Fixierung auf die westeuropäische Kunstszene fest (Drews 1975: 303).

14 Zur Rezeption Whitmans in Böhmen vgl. Drews 1983: 147-152.

$15 \mathrm{Zu}$ Apollinaire äußerte sich Teige darüber hinaus 1928 erneut und in intensiver Weise in seinem Manifest poetismu (Teige 1966: 332-336 bzw. 348-350), daneben aber auch in mehreren eigenen Beiträgen speziell zu dem französischen Autor, etwa ebenfalls 1928 anlässlich des zehnten Todestags des Dichters in Guillaume Apollinaire a jeho doba (Teige 1966: 371-404). - Zur Rezeption Apollinaires in den westslawischen literarischen Avantgardebewegungen vgl. grundlegend Winczer 2000: 38-90.

16 Âhnlich wie zu Apollinaire, so äußerte sich Teige auch eigens zu Marinetti; nach dessen Besuch in Prag im Dezember 1921 veröffentlichte Teige noch Mitte Jänner des darauf folgenden Jahres den Beitrag F. T. Marinetti a futurismus, 1925 dann Futurismus a italská moderna und 1929 schließlich die Abhandlung F. T. Marinetti-italská moderna + světový futurismus (Teige 1966: 539). - Zur Aufnahme des italienischen Futurismus in Böhmen vgl. Drews 1983: 184 ff.; Drews kommt zum Schluss, dass es insgesamt nur sehr wenige direkte Berührungspunkte zwischen der tschechischen Avantgarde und dem Futurismus gegeben habe (187).

17 Dirk Uffelmann nennt (insbesondere für Teiges zweites poetistisches Manifest aus dem Jahr 1928) die französische Kultur als entsprechende „Schlüsseltradition“ (Uffelmann 1997: 394). 
europäische kulturelle Tradition mit großer provokativer Geste für obsolet zu erklären, vom tschechischen Poetisten Teige dagegen wird diese Achse passagenweise zwar durchaus ähnlich funktionalisiert, daneben aber auch zugunsten einer panchronischen Simultanität aufgehoben, durch die Homer und Charlie Chaplin plötzlich unmittelbar Seite an Seite und nur durch einen Punkt graphisch voneinander getrennt zu stehen kommen (126) - ein Verfahren übrigens, das in Verbindung mit der von Teige postulierten Diesseitigkeit der Kunst von ferne an die Programmatik und poetische Praxis des russischen Akmeismus mit Autoren wie Osip Mandel'štam erinnert, thematisierte letzterer doch in seinen Gedichten 1913 in ähnlich spielerischer Weise wie später dann die Poetisten Nezval oder Seifert etwa den Sport im Allgemeinen, das Tennis- und Fußballspiel, das Kino oder eine American Bar (Mandel'štam 1993: 87-95).

Hält man nun die drei erwähnten Programmtexte von Bruno Jasieński, Ladislav Novomeský und Karel Teige gegeneinander, so treten über das Phänomen der Offenheit Gemeinsamkeiten wie Unterschiede in der Programmatik der tschechischen, slowakischen und polnischen Avantgarde deutlich hervor, wobei der punktuelle Charakter der vorliegenden Analyse natürlich stets mitreflektiert werden muss. Meint Offenheit die von der historischen Avantgarde angestrebte Erweiterung der poetischen Möglichkeiten und die Dehierarchisierung tradierter kultureller Wertsetzungen, so erweisen sich alle drei Programmtexte etwa in der Forderung, die Straße als neuen Handlungsraum der Kunst zu etablieren, als in hohem Ausmaß analog. Bedeutet Offenheit dagegen das Setzen von interkulturellen Verweisen und die dadurch generierte Integration der eigenen Positionen in einen weiter reichenden Rahmen gesamteuropäischer Kultur, so unterscheiden sich die drei Texte wiederum ebenso deutlich voneinander. $\mathrm{Ob}$ mit diesem (hier lediglich an drei Einzeltexten) festgemachten Interferieren von Gemeinsamkeiten und Unterschieden auch die wechselseitigen Verbindungen der drei westslawischen Avantgardebewegungen insgesamt paradigmatisch auf einen Nenner zu bringen sind, kann an dieser Stelle naturgemäß nicht entschieden werden. In den Raum gestellt sei diese Möglichkeit aber allemal.

\section{Literatur}

Asholt - Fähnders 1995: Wolfgang Asholt, Walter Fähnders (Hrsg.), Manifeste und Proklamationen der europäischen Avantgarde (1909-1938), Stuttgart - Weimar

Bahr 1968: Hermann Bahr, Zur Überwindung des Naturalismus. Theoretische Schriften 1887-1904. Hrsg. v. Gotthart Wunberg, Stuttgart u. a.

Benjamin 2003: $\quad$ Walter Benjamin, Das Kunstwerk im Zeitalter seiner technischen Reproduzierbarkeit. Drei Studien zur Kunstsoziologie, Frankfurt am Main

Boden 2005: $\quad$ Doris Boden, Pulsation versus Chiasmus: Laco Novomeskýs Lyriksammlung „Romboid“ und der tschechische Poetismus, in: Alfrun Kliems (Hrsg.), Slowakische Kultur und Literatur im Selbst- und Fremdverständnis. Ludwig Richter zum 70. Geburtstag, Stuttgart, $129-150$ 
Brousek 1975: $\quad$ Marketa Brousek, Der Poetismus. Die Lehrjahre der tschechischen Avantgarde und ihrer marxistischen Kritiker, München

Brzozowski 1973: $\quad$ Stanisław Brzozowski, My młodzi, in: Maria Podraza-Kwiatkowska (Hrsg.), Programy i dyskusje literackie okresu Młodej Polski, Wroclaw u. a., $128-139$

Bürger 2007: $\quad$ Peter Bürger, Theorie der Avantgarde, Frankfurt am Main (14. Aufl.)

Bydžovská 1999: $\quad$ Lenka Bydžovská, The Avant-Garde Ideal of Poiēsis: Poetism and Artificialism during the Late 1920s, in: Eric Dluhosch, Rostislav Švácha (Hrsg.), Karel Teige: l'enfant terrible of the Czech modernist avantgarde, Cambridge (Mass.) - London, 46-63

Česká moderna 1950: Ceská moderna, in: Soubor díla F. X. Šaldy 11, Praha, 361-363

Drews 1975:

Peter Drews, Devětsil und Poetismus. Künstlerische Theorie und Praxis der tschechischen literarischen Avantgarde am Beispiel Vítězslav Nezvals, Jaroslav Seiferts und Jiř́ Wolkers, München

Drews 1983: $\quad$ Peter Drews, Die slawische Avantgarde und der Westen. Die Programme der russischen, polnischen und tschechischen literarischen Avantgarde und ihr europäischer Kontext, München

Fabian 2001: Jeanette Fabian, Karel Teige a evropská moderna, Literární Archiv 32-33, 63-91

Foucault 1997: $\quad$ Michel Foucault, Die Ordnung des Diskurses. Aus dem Französischen von Walter Seitter. Mit einem Essay von Ralf Konersmann, Frankfurt am Main (13.-15. Tsnd.)

Grübel 1998: $\quad$ Rainer Grübel, Literaturersatz, handgreifliche Kunst oder Vor-Schrift? Diskurspragmatik und Bauformen, Axiologie und Intentionalität literarischer Deklarationen, Manifeste und Programme der russischen Moderne (1893-1934), in: Hubert van der Berg, Ralf Grüttemeier (Hrsg.), Manifeste: Intentionalität, Amsterdam, 161-192

Hansen-Löve 1975: Christiana Hansen-Löve, Die tschechische Avantgarde-Kunst der Zwanzigerjahre. Der Poetismus, Wien (Diss.)

Jasieński 1969: Bruno Jasieński, Do narodu polskiego. Manifest w sprawie natychmiastowej futuryzacji życia, in: Andrzej Lam (Hrsg.), Polska awangarda poetycka. Programy lat 1917-1923, t. II, Kraków, 208-214

Krzychylkiewicz 2006: Agata Krzychylkiewicz, The grotesque in the works of Bruno Jasienski, Bern

Lotman 1973: $\quad$ Jurij M. Lotman, Die Struktur des künstlerischen Textes. Hrsg. v. Rainer Grübel, Frankfurt am Main

Mandel'štam 1993: $\quad$ Osip Mandel'štam, Sobranie sočinenij v 4-ch tomach, t. 1, Moskva

Müller 1978:

Vladimír Müller, Der Poetismus. Das Programm und die Hauptverfahren der tschechischen literarischen Avantgarde der zwanziger Jahre, München

Novomeský 1970: Ladislav Novomeský, Manifesty a protesty. Výber zo statí a príspevkov o kultúre a umení 1924-1937, Bratislava

Novomeský 1971: $\quad$ Laco Novomeský, Basnické dielo 1, Bratislava

Patera 1974: $\quad$ Ludvík Patera, Ladislav Novomeský a česká literatúra, in: Karol Rosenbaum (Hrsg.), Ladislav Novomeský 1904 - 1974, Martin, 187-202

Poščečina 1924: $\quad$ Poščečina obščestvennomu vkusu, in: N. L. Brodskij, N. P. Sidorov (Hrsg.), Ot simvolizma do „Oktjabrja“, Moskva, 99-100

Przesmycki-Miriam 1973: Zenon Przesmycki, Nasze zamiary, in: Maria Podraza-Kwiatkowska (Hrsg.), Programy i dyskusje literackie okresu Młodej Polski, Wrocław u. a., 183-188

Simonek 2005: $\quad$ Stefan Simonek, Europäisierung als Verheißung oder Bedrohung? Ein vergleichender Blick auf Programmtexte der Moderne in den ostslawischen Literaturen, Wiener Slavistisches Jahrbuch 51, 197-205

Stern 1969: Anatol Stern, Bruno Jasieński, Warszawa

Sus 1964: Oleg Sus, První manifest poetismu Karla Teiga, Slovenská literatúra XI, 367-382 
Teige 1966:

Karel Teige, Výbor z díla I: Svět stavby a básně. Studie z dvacátých

Uffelmann 1997: let, Praha

Dirk Uffelmann, Maximální funkčnost. Architektur und Poesie in der Theorie Karel Teiges 1924-1930 - Ein Baustein zur Genealogie totalen Denkens, Österreichische Osthefte 39, 381-410

Winczer 2000:

Pavol Winczer, Súvislosti v čase a priestore. Básnická avantgarda, jej prekonávanie a dedičstvo (Čechy, Slovensko, Pol'sko), Bratislava

Winczer 2001:

Pavol Winczer, Die slowakische Avantgarde im europäischen Umfeld, in: Reinhard Lauer (Hrsg.), Die literarische Avantgarde in Südosteuropa und ihre politische und gesellschaftliche Bedeutung, München, 4976

Zusi 2004:

Peter A. Zusi, The Style of the Present: Karel Teige on Constructivism and Poetism, Representations 88, 102-124

(http://caliber.ucpress.net/doi/pdf/10.1525/rep.2004.88.1.102, abgerufen am 18. 5. 2010)

A b stract: Openness as a literary device in the manifestos of the Czech, Polish and Slovak interwar avant-garde. The essay offers an analysis of the discursive strategies that create the notions of openness and closeness in the essential manifestos of the Czech, Slovak and Polish avant-garde of the 1920s. A comparison of Bruno Jasieński's, Karel Teige's and Ladislav Novomeský's manifestos shows up the different connections between openness and closeness in these texts: with regard to closeness, all three authors evoke in a similar way a radical overcoming of literary tradition in creating a distinct form of speech in the name of their own avant-garde groups. Contradicting this rhetorical evocation of closeness, all three manifestos are characterized by a significant longing for (spatial) openness, to be achieved by rejecting the traditional closed spaces of an elitist form of high culture, such as a museum, a library or a concert hall, in favour of dynamic, popular and essentially open forms of art, which move the location of culture out of these traditional closed rooms and into the street. This specific kind of openness can be found in all manifestos, whereas Teige, Jasieński and Novomeský each interpret openness as a cultural notion in a different way. In Novomeský's text, for example, we will not find a single reference to a foreign writer or artist; Jasieński, in his futurist manifesto, opposes the Polish cultural tradition to European cultural values; and Karel Teige, finally, understands poetism as an equal part of European culture and, therefore, in his manifesto, refers to Whitman, Apollinaire and several other European writers. As a result of this, openness as a spatial notion is represented in all the three texts, while openness to European culture, on the other hand, is treated in the Czech, the Polish and the Slovak manifestos in different ways, ranging from absence and contradiction to full participation in European culture.

K e y w o rd s: Czech, Polish, Slovak avant-garde, manifesto

Stefan Simonek

Institut für Slawistik der Universität Wien

Universitätscampus AAKH, Hof 3

Spitalgasse 2, 1090 Wien, Österreich

stefan.simonek@univie.ac.at 